\title{
Digital Subtraction Angiography of Anterior Cerebral Artery Complex in Indian Population at Two Tertiary Super-Specialty Hospitals
}

\author{
Md. Amir Hossain', Shakir Husain², Md. Shohidul Islam³ ${ }^{3}$ Muhammed Abdul Momen Khan ${ }^{4}$ \\ ${ }^{1}$ Assistant Professor, Department of Interventional Neurology, National Institute of Neurosciences \& Hospital, Dhaka, \\ Bangladesh; ${ }^{2}$ Director of Neurointervention, Max Super-specialty Hospital, New Delhi, India \& Visiting Professor of \\ Neurointervention, National Institute of Neurosciences \& Hospital, Dhaka, Bangladesh; ${ }^{3}$ Assistant Professor of \\ Neurology, Officer on Special Duty (OSD), Directorate General of Health Services, Ministry of Health \& \\ Family Welfare, Dhaka, Bangladesh; ${ }^{4}$ Assistant Professor, Department of Interventional Neurology, \\ National Institute of Neurosciences \& Hospital, Dhaka, Bangladesh
}

[Received: 21 June 2017; Revised: 6 November 2017; Accepted: 11 December 2017; Published: 1 January 2018]

\begin{abstract}
Background: Cerebral circulation has different variations in blood supply. Anterior cerebral artery is an important terminal branch of internal carotid artery. It forms the anterior component of circle of Willis along with the anterior communicating artery. The knowledge of anatomical variations in anterior cerebral artery is of considerable help to clinicians. Objective: The purpose of the study is to find out common anatomical variations of anterior cerebral artery complex by digital subtraction angiography in Indian population. Methodology: This descriptive cross-sectional study was conducted in the Neurointervention department of Max Super-specialty Hospital, New Delhi and Neo Multi-specialty Hospital, Noida, India during July 2016 to December 2016 for a period of six (06) months. Patients admitted in the Neurointervention department for digital subtraction angiography (DSA) were included in this study. Sampling technique was purposive. The angiogram machine was SIEMENS Artis Zee system and framing rate was $4 \mathrm{f} / \mathrm{sec}$. Morphology and variations of the anterior cerebral arteries and the anterior communicating artery were studied in 75 patients undergone cerebral DSA. Results: Variations were found in $48 \%(n=36)$. Variations of the segments in relation with size, course, communications and terminations of the anterior cerebral artery (ACA) were noted. These were divided into different groups like hypoplasia, aplasia, duplication and fenestrations. Hypoplasia/Aplasia of proximal anterior cerebral artery (A1) was 13.3\% in right side and 5.3\% in left side. Anterior communicating artery (AComA) was found absent in $10.7 \%$ and fenestration in $12 \%$. Callosomarginal artery was found absent in $1.3 \%$ in right side and $5.3 \%$ in left side. In right callosomarginal artery $6.8 \%$ had abnormal origin and $4.2 \%$ abnormal in left side. Pericallosal artery was present $100 \%$ on both sides. Conclusion: Variations of anterior cerebral artery complex anatomy is found common in Indian population. Among them hypoplasia/aplasia is most common. [Journal of National Institute of Neurosciences Bangladesh, 2018;4(1): 12-17]
\end{abstract}

Keywords: Anterior cerebral artery complex; variations; hypoplasia; aplasia; fenestration

Correspondence: Dr. Md. Amir Hossain, Assistant Professor, Department of Interventional Neurology, National Institute of Neurosciences \& Hospital, Sher-E-Bangla Nagar, Agargaon, Dhaka-1207, Bangladesh; Email: amir.dr@gmail.com; Cell: +8801711382650 ;

Conflict of Interest: The authors declare that they have no competing interest.

Contributions to Authors': Hossain MA \& Husain S involved in the protocol preparation, concept of protocol, procedure and collection of data upto report writing. Islam MS, Khan MAM had revised the manuscript. All the authors have read and approved the final version of the manuscript.

Funding: This research project was not funded by any group or any institute on.

How to cite this article: Hossain MA, Husain S, Islam MS, Khan MAM. Digital Subtraction Angiography of Anterior Cerebral Artery Complex in Indian Population at Two Tertiary Super-Specialty Hospitals. J Natl Inst Neurosci Bangladesh, 2018;4(1): 12-17

Copyright: (C2018. Hossain et al. Published by Journal of National Institute of Neurosciences Bangladesh. This article is published under the Creative Commons CC BY-NC License (https://creativecommons.org/licenses/by-nc/4.0/). This license permits use, distribution and reproduction in any medium, provided the original work is properly cited, and is not used for commercial purposes.

\section{Introduction}

The cerebral cortex is primarily supplied by the anterior, middle and posterior cerebral arteries. The anterior communicating artery complex consists of two anterior cerebral arteries (ACA), one anterior communicating artery (AComA) and two recurrent arteries of Heubner ${ }^{1-2}$. The anatomy of the anterior cerebral artery (ACA) is variable and the description of the ACA and its branches 
is complicated. Different authors have described the segments of the ACA differently. It can be divided into proximal or pre-communicating and distal or post-communicating segments $\mathrm{s}^{3-4}$. The pericallosal artery is distal to the A1 segment and consists of several segments that can be divided according to its relationship with the corpus callosum. The A2 segment (infracallosal section) runs vertically from the AComA to the genu of the corpus callosum. The A3 segment (precallosal part) curves around the genu, and the A4 segment (supracallosal section) usually runs in the callosal sulcus and almost reaches the splenium ${ }^{5-6}$.

The A5 segment (cortical branches) varies considerably; for this reason it is difficult to describe a standard arterial pattern. The two basic configurations of the ACA are determined by the presence or absence of the $\mathrm{CmA}^{6-7}$. Some authors refer to the A1 segment as the ACA and the artery distal to the AComA as the pericallosal artery ${ }^{8}$. The ACA complex has a strong clinical importance. It is the most common site of intracranial aneurysm. Despite its considerable significance, little is known about the anatomical variations of the ACA complex. Many anomalies such as aplasia, hypoplasia, duplication or fenestration of ACA segments and AComA have been described. Authors used various methods such as digital subtraction angiography (DSA), computed tomography angiography (CTA), magnetic resonance angiography (MRA), cadaveric dissection or intraoperative observations to study the anterior cerebral circulation of brain. However those studies have a number of limitations. Firstly, they are focused on patients with intracranial aneurysms, and not healthy subjects?. Secondly, the authors base their conclusions on a relatively small study group, rarely exceeding 100 patients. Thirdly, their observations are often limited to the anomalies of the A1 segment (most commonly associated with AComA aneurysms) regardless of AComA and A2 and other segment anomalies. Complete study of anterior cerebral artery complex is lacking in the population of Indian subcontinent. Digital subtraction angiography is now-a-days becoming popular minimally invasive angiographic study method for cerebral vessels. Most of the pathologies of cerebral vasculature can be explored and treated by Neurointervention methods ${ }^{10}$. The development of Neurointervention is progressively increasing in the field of management of vascular pathologies in brain.

So complete understanding by the help of digital subtraction angiography of ACA complex is very important for the population of Indian subcontinent. The results of such studies would be useful to make plan in neurointerventional procedures and surgical approaches, and would allow avoiding any unexpected anatomical variations during treatment of AComA aneurysms. The aim of this study was to see the common variations of ACA complex configurations by using digital subtraction angiography (DSA).

\section{Methodology}

This descriptive cross-sectional study was conducted in the Department of Neurointervention of Max Super-specialty Hospital, New Delhi, India and Neo Multi-specialty Hospital, Noida, Uttar Pradesh, India during July 2016 to December 2016 for a period of six (06) months. Patients admitted in the Neurointervention department for digital subtraction angiography (DSA) were included in this study. Sampling technique was purposive. The angiogram machine was SIEMENS Artis Zee system and framing rate was $4 \mathrm{f} / \mathrm{sec}$. Angiograms with gross pathology like arteriovenous malformations (AVM) in anterior circulation were excluded from this study. Anterior part of the circle of Willis was studied. Morphological variations, branching pattern and course of the ACA and AComA were observed. Variations of the size, course, segments, communications and terminations of the anterior cerebral artery complex were noted. These variations were divided into different groups like hypoplasia, aplasia, and duplication of pre-communicating segment of ACA (A1), double AComA, fenestrations, azygos ACA and variation in the A2 segment of ACA in its terminal branches. Origin of callosomarginal artery and the course of pericallosal artery were noted and pictures were drawn in data collection sheet. All collected data were checked, edited and analyzed by using computer based SPSS software version 16.0. Data were presented by frequency distribution and percentage.

\section{Results}

A total of 75 patients admitted in the Neurointervention department for digital subtraction angiography (DSA) included in this study. Out of the 75 patients, majority were belonged to over the age 40 years which was

Table 1: Socio-demographic characteristics of the study patients $(\mathrm{n}=75)$

\begin{tabular}{llcc}
\hline \multicolumn{2}{l}{ Characteristics } & Frequency & Percent \\
\hline Sex & Male & 53 & 70.7 \\
& Female & 22 & 29.3 \\
Age & $\leq 40$ & 17 & 22.7 \\
& $>40$ & 58 & 77.3 \\
\hline
\end{tabular}


$58(77.3 \%)$ cases and 40 or less than 40 years were $17(22.7 \%)$ cases. It was observed that $53(70.7 \%)$ cases were male and 22(29.3\%) cases were female. Male to female ratio was 2.4:1 (Table 1).

Morphologic variations like Aplasia/hypoplasia/ fenestration in A1 or AcomA or absent/abnormal origin of callosomarginal artery were present in $36(48 \%)$ of cases. Variations related with the A1 segment of ACA are agenesis, hypoplasia and duplication. Hypo- plastic/ under-developed A1/Aplastic segment were present in 14 cases. It was seen in $10(13.3 \%)$ on the right and 4 (5.3\%) on the left A1 segment Azygos anterior cerebral artery was found in $2(2.7 \%)$ cases. Triple A2 segment was found in $1(1.3 \%)$ case (Table 2$)$.

Table 2: Morphologic variation of A1 segment of ACA on both sides $(\mathrm{n}=75)$

\begin{tabular}{lcc}
\hline Right A1 & Frequency & Percent \\
\hline Normal & 65 & 86.7 \\
Hypoplasia/Aplasia & 10 & 13.3 \\
Left A1 & & \\
Normal & 71 & 94.7 \\
Hypoplasia/Aplasia & 4 & 5.3 \\
\hline
\end{tabular}

All possible forms of abnormalities of AComA were present in $19(25.4 \%)$ of cases and normal in 56 $(74.6 \%)$ cases. Fenestration of AcomA was seen in 9 $(12 \%)$ cases, Hypoplastic/Absent AComA was seen in $8(10.7 \%)$ cases. Other abnormalities like duplication were present $2(2.7 \%)$ cases (Table 3$)$.

Table 3: Morphologic variation of AComA of ACA complexes $(\mathrm{n}=75)$

\begin{tabular}{lcc}
\hline AComA & Frequency & Percent \\
\hline Normal & 56 & 74.6 \\
Fenestration & 9 & 12 \\
Hypoplasia/Aplasia & 8 & 10.7 \\
Others & 2 & 2.7 \\
\hline
\end{tabular}

Callosomarginal artery was absent in $1(1.3 \%)$ in right side and $4(5.3 \%)$ in left side. Out of them $5(6.8 \%)$ in

Table 4A: Morphologic variation of origin of Right Callosomarginal artery $(\mathrm{n}=75)$

\begin{tabular}{lcc}
\hline Right CmA Artery & Frequency & Percent \\
\hline Present & 74 & 98.7 \\
Absent & 1 & 1.3 \\
Origin & & \\
Normal & 69 & 93.2 \\
Abnormal & 5 & 6.8 \\
\hline
\end{tabular}

callosomarginal arteries $=\mathrm{CmA}$ right side and $3(4.2 \%)$ in left side were abnormal in origin. Pericallosal artery was found in all cases on both sides in this study (Table 4A, 4B).

Table 4B: Morphologic variation of origin of Left Callosomarginal artery $(n=75)$

\begin{tabular}{lcc}
\hline Left CmA Artery & Frequency & Percent \\
\hline Present & 71 & 94.7 \\
Absent & 4 & 5.3 \\
Origin & & \\
Normal & 68 & 95.8 \\
Abnormal & 3 & 4.2 \\
\hline
\end{tabular}

callosomarginal arteries $=\mathrm{CmA}$

\section{Discussion}

Multiple variations in anatomy of anterior cerebral artery complex like agenesis, hypoplasia, fenestrations resulting in defective circulation has been reported in the different publications ${ }^{11}$. If the artery on one side is narrowed, the vascular insufficiency is compensated by crossing over by opposite side artery, or by giving branches that cross over to the other side. It indicates that the circle of Willis offers a potential shunt in abnormal conditions such as occlusions and spasms. In normal circumstances it is not an equalizer and distributer of blood from different sources ${ }^{12}$.

Majority $(77.3 \%)$ of the study subject was over 40 years of age, and less than 40 years is $22.7 \%$. Among them male were $70.7 \%$ and female were $29.3 \%$, and male to female ratio were 2.4:1. Morphological variations like Aplasia/hypoplasia/fenestration in A1 or AcomA or absent/abnormal origin of callosomarginal artery was present in 36(48\%) of cases. In Text book of Practical Neuroangiography by Pearse Morris, third edition showed the range of variation between $11.0 \%$ to $43.0 \%{ }^{2}$. In a cadaveric study Gunnal et $\mathrm{al}^{13}$ found total variation up to $31.3 \%$ cases.

Variations related with the A1 segment of ACA are agenesis, hypoplasia and duplication ${ }^{14}$. Hypoplastic/ under-developed A1/Aplastic segment were present in 14 cases. It was seen in $10(13.3 \%)$ on the right and $4(5.3 \%)$ on the left A1 segment. In previous cadaveric study by Riggs and Rup ${ }^{4}$ showed $7.0 \%$ A1 hypoplasia. Pai et $\mathrm{al}^{5}$ found no hypoplasia or aplasia. But Piganiol et $\mathrm{al}^{6}$ found $2.1 \%$ and Macchi et $\mathrm{al}^{7}$ found it $0.7 \%$. 3DCTA study showed $10 \%$ hypoplasia/apasia in a series $^{15}$. In general, our study shows a higher percentage of variations. Hypoplasia/Aplasia is more common in right side than the left side $(13.3 \%$ vs $5.3 \%)$. 


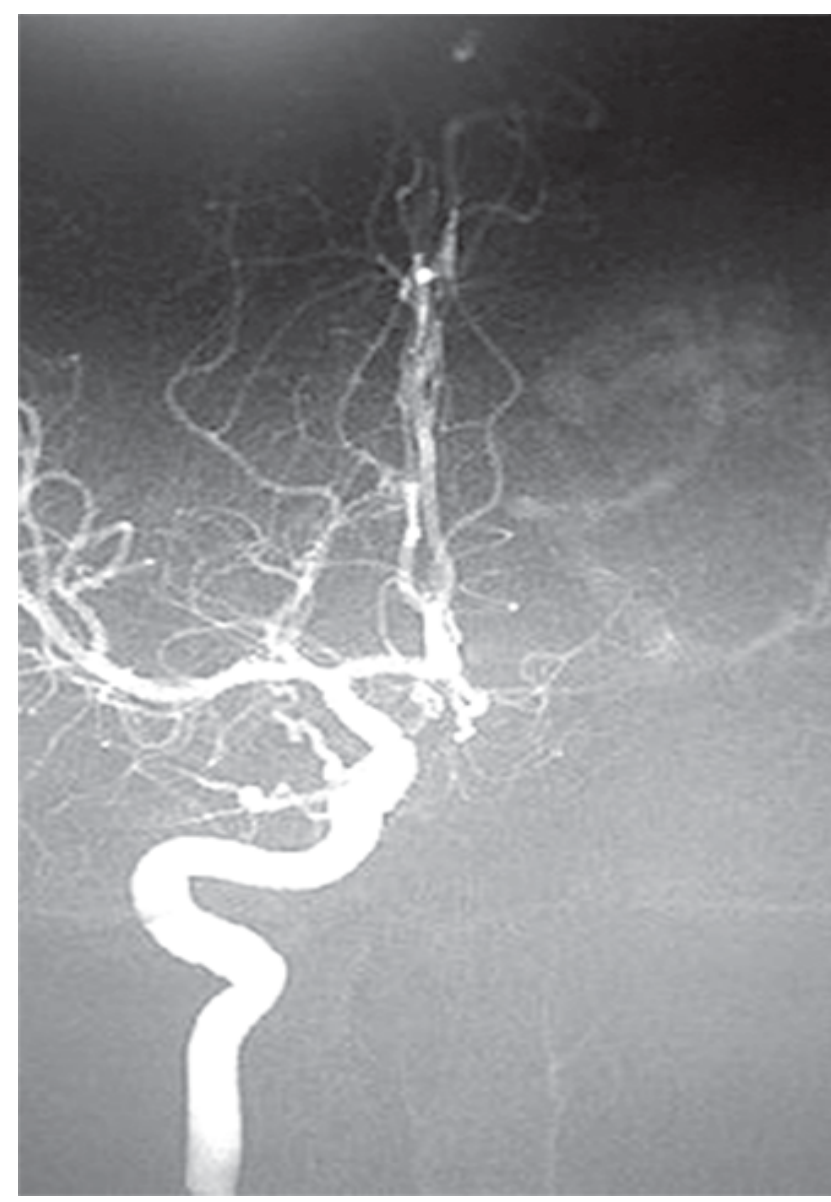

Figure 1: Azygous A2 with left hypoplastic A1

All possible forms of abnormalities of AComA were present in 19(25.4\%) of cases and normal in 56(74.6\%) cases. Fenestration of AcomA was seen in $9(12 \%)$ cases, Hypoplastic/ Absent AComA was seen in $8(10.7 \%)$ cases. Other abnormalities like duplication were present $2(2.7 \%)$ cases. Autopsy studies described the frequency of fenestrations in the anterior circulation in up to $64.4 \%$. Investigations that used three-dimensional digital subtraction angiography (DSA) found a greater incidence of fenestrations in the anterior cerebral circulation, which was $27 \%$ for aneurysm patients and $22 \%$ for patients without aneurysm $^{15-16}$. On the other hand, Sanders et $\mathrm{al}^{16}$ studying 5,190 cerebral angiograms found only 3 fenestrations in the AComA complex. A study by Boz ek et $\mathrm{al}^{3}$ using CTA described the incidence of AComA complex fenestrations to be $1.75 \%$. Zhao et a ${ }^{14}$, Saidi et $\mathrm{al}^{17}$ in cadaveric study found fenestration $0,8 \%, 1.2 \%$ and $26 \%$ respectively. These findings emphasis the fact that two-dimensional imaging is not a suitable tool for detecting intracranial arterial fenestrations. So, the most common anomaly of the anterior cerebral circulation was fenestrated AComA.
Again Cadaveric studies show a vast range of AComA hypoplasia frequency from 9.15 to $30.0 \%{ }^{12}$. In addition, aplastic AComA is a rare autopsy finding, found only in $1.8 \%$ of studied subjects ${ }^{13-18}$. This phenomenon can be explained by the fact that hypoplastic arteries may not be hemodynamically efficient, therefore are not visible in angiographic studies and thus are considered to be aplastic ${ }^{19,15}$. On the other hand, autopsy findings always visualize the artery trunk, even when contrast flow would not be possible. Li et a $1^{8}$ using CTA found aplastic AComA's in $9.38 \%$ of study subjects. AComA $3 \mathrm{D}$ imaging provides the excellent quality data, but unfortunately it is rarely available in everyday clinical practice $^{20,16}$. Pai et al ${ }^{5}$ showed $20 \%$ aplastic/hypoplastic AComA.

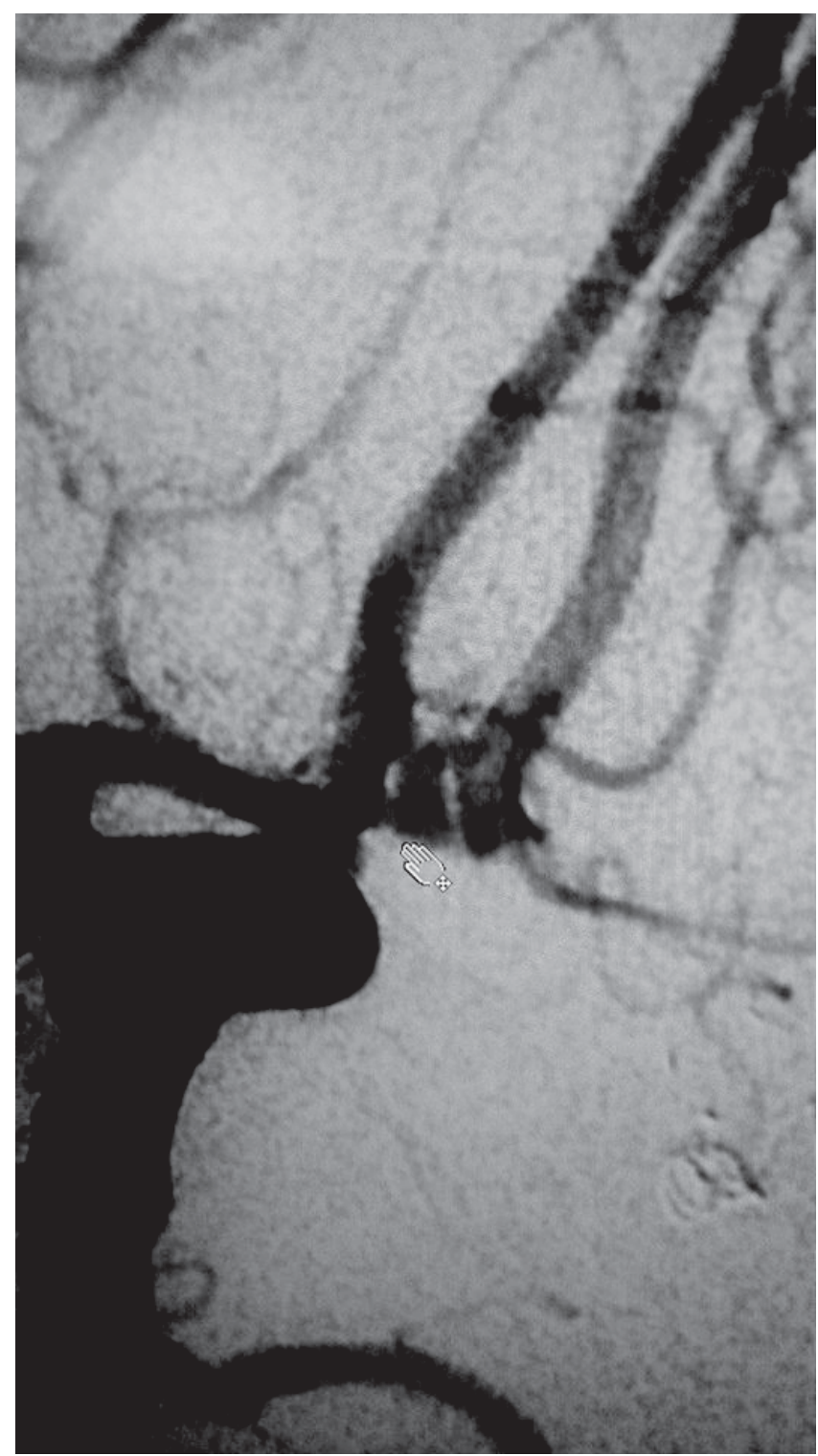

Figure 2: Fenestration of AComA 
Azygos anterior cerebral artery was found in 2 (2.7\%) cases. In text book Practical Neuroangiography by Pearse Morris showed that incidence of azygos ACA is only $0.3 \% 2$. Schik et a $1^{17}$, Dietrich et al18 and Baptista et $\mathrm{al}^{22}$ found $1.1 \%, 0.5-5 \%, 0-5 \%$ and $0.1-5 \%$ respectively. But some study like Osborn et $\mathrm{al}^{24}$ showed this value very high $(10 \%)$. In this study we found one $(1.3 \%)$ case of a triple A2 segment. Most authors identify the triple A2 segment as a persistent median artery of corpus callosum, a remnant of embryological cerebral circulation ${ }^{11}$. MRA studies show that the frequency of a triple A2 segment ranges between 0.4 and $3.03 \%{ }^{23-25}$. Usually a triple A2 is an incidental finding. Sun et $\mathrm{a}^{16}$ reported a very interesting case of a triple A2 segment associated with the presence of an aneurysm. We have not found such pathology in our study.

Callosomarginal artery was absent in $1(1.3 \%)$ in right side and $4(5.3 \%)$ in left side. Out of them 5(6.8\%) from right side and 3(4.2\%) from left side were abnormal in origin. Callosomarginal artery usually originates at the top of the knee of corpus callosum. But very early origin or late origin are also seen. Abnormal origin may be associated with aneurysm formation. The $\mathrm{CmA}$ is also not always present and therefore it is preferable to classify the pericallosal artery as the segment distal to the $\mathrm{AComA}^{25,17}$. The CmA has been observed in $40.0 \%$ to $93.4 \%$ of specimens ${ }^{21-29}$. The variability of the absence or presence of the $\mathrm{CmA}$ is due to the different definitions used for this artery by different authors ${ }^{26-30}$. The $\mathrm{CmA}$ is the largest branch of the pericallosal artery that has been defined as the artery that runs near the cingulate sulcus and gives off two or more cortical branches. But this is problematic since there can occasionally be more than one artery that arises from the pericallosal artery, run in the cingulate sulcus and give rise to a number of cortical branches ${ }^{30}$. Ugur et al ${ }^{30}$ proposed a new classification system. The CmA was either defined as typical, atypical or absent. An atypical CmA was observed when there was only a very short artery coursing in the cingulate sulcus. Two symmetrical callosomarginal arteries can also be present in the same hemisphere. A typical CmA has a longer course compared to the two symmetrical atypical callosomarginal arteries and usually originates from the A3 segment. Ugur et $\mathrm{al}^{30}$ observed typical, atypical or absent CmA's in $49.0 \%, 34.0 \%$ and $17.0 \%$ respectively.

\section{Conclusion}

Anterior cerebral artery complex is a common place for anatomical variations and this has a relation with aneurysm formations. Morphology and variations of the anterior cerebral artery complex were studied in Indian patients who had undergone digital subtraction angiography (DSA). Among them a large number had different forms of variations. Hypoplasia/Aplasia of proximal anterior cerebral artery (A1) was most common anatomical variation and that was most common in right side. Other variations were also present. This study was performed in a small number of subjects. Multicenter studies are necessary to reach a satisfactory conclusion about the complete understanding of anatomy of anterior cerebral artery complex in this population.

\section{References}

1. Krayenbuhl HA, Yasargil MG. Cerebral angiography. 2nd Ed. New York: Thieme Medical Publishers Inc, 1982:91-3

2. Morris P. Practical Neuroangiography. 3rd Ed. Philadelphia: Lippincott Williams \& Wilkins, 2013:161-74

3. Boz ek P, Pilch-Kowalczyk J, Kluczewska E, Zymon-Zagórska A. Detection of cerebral artery fenestrations by computed tomography angiography. Neurol Neurochir Pol. 2012; 46:239-244

4. Riggs HE, Rupp C. Variations in form of Circle of Willis. Arch Neurol 1963; 8(1):8-14

5. Pai SB, Kulkarni RN, Varma RG. Microsurgical anatomy of the anterior cerebral artery-Anterior communicating artery complex: An Indian study. Neurol Asia 2005; 10:21-8

6. Piganiol G, Toga M, Paillas J. L'arterecommuni-canteanterieure, Etude embriologiqueet anatomique. Neurochirurgie 1960; 6:3-19 7. Macchi C, Catini C, Federico C, Gulisano M, Pacini P, Cecchi F. Magnetic resonance angiographic evaluation of circulusarteriosuscerebri (circle of Willis): a morphologic study in 100 human healthy subjects. Ital J Anat Embryol 1996; 101:115-23 8. Li Q, Li J, Lv F, Li K, Luo T, Xie P. A multidetector CT angiography study of variations in the circle of Willis in a Chinese population. J Clin Neurosci 2011;18:379-383

9. Maga P, Tomaszewski KA, Pasternak A, Zawilinski J, Tomaszewska R, Gregorczyk-Maga I et al. Extra- and intracerebral course of the recurrent artery of Heubner. Folia Morphol (Warsz) 2013; 72:94-99

10. Maga P, Tomaszewski KA, Skrzat J, Tomaszewska IM, Iskra T, Pasternak A, et al. Microanatomical study of the recurrent artery of Heubner. Ann Anat 2013; 195:342-350

11. Gomes FB, Dujovny M, Umansky F, Berman SK, Diaz FG, Ausman JI, Mirchandani HG, Ray WJ. Microanatomy of the anterior cerebral artery. Surg Neurol 1986; 26(2):129-141

12. Compton MR. The pathology of ruptured ACA aneurysms. Lancet 1962; 2:421-38

13. Gunnal SA, Wabale RN, Farooqui MS. Variations of anterior cerebral artery in human cadavers: Neurology Asia 2013;18(3):249-259

14. Zhao HW, Fu J, Lu ZL, Lü HJ. Fenestration of the anterior cerebral artery detected by magnetic resonance angiography. Chin Med J (Engl) 2009; 259

15. Parmar H, Sitoh YY, Hui F. Normal variants of the intracranial circulation demonstrated by MR angiography at $3 \mathrm{~T}$. Eur J Radiol 2005; 56:220-228

16. Sanders WP, Sorek PA, Mehta BA. Fenestration of intracranial arteries with special attention to associated aneurysms and other anomalies. AJNR Am J Neuroradiol 1993; 14:675-680 
17. Saidi H, Kitunguu P, Ogengo JA. Variant anatomy of the anterior cerebral artery in Adult Kenyans. Afr J Neurol Sci 2008; 27:97-105

18. Sun C, Xv ZD, Yuan ZG, Wang XM, Wang LJ, Liu C. MSCT diagnosis of aneurysms associated with an unusual variant: atypical triplication anterior cerebral artery. Surg Radiol Anat 2012; 34:777-780

19. Kakou M, Destrieux C, Velut S. Microanatomy of the pericallosal arterial complex. J Neurosurg 2000;93:667-675

20. Dietrich W, Reinprecht A, Gruber A, Czech T. De novo formation and rupture of an azygospericallosal artery aneurysm. Case report. J Neurosurg 2000; 93:1062-4

21. Stefani MA, Schneider FL, Marrone AC, Severino AG, Jackowski AP, Wallace MC. Anatomic variations of anterior cerebral artery cortical branches. Clin Anat 2000;13:231-6

22. Baptista AG. Studies on the arteries of the brain. II. The anterior cerebral artery: Some anatomic features and their clinical implications. Neurology 1963;13:825-35

23. Zurada A, Gielecki J, Shane Tubbs R, Loukas M, Maksymowicz W, Chlebiej M. Detailed 3D-morphometry of the anterior communicating artery: potential clinical and neurosurgical implications. Surg Radiol Anat 2011; 33:531-538

24. Kedia S, Daisy S, Mukherjee KK, Salunke P, Srinivasa R, Narain MS. Microsurgical anatomy of the anterior cerebral artery in Indian cadavers. Neurol India 2013;61(2):117-121

25. Osborn AG. Diagnostic Cerebral Angiography. 2nd Ed. Philadelphia, PA: Lippincott Williams \& Wilkins. Neurology 1999:34-9

26. Perlmutter D, Rhoton AL. Microsurgical anatomy of the distal anterior cerebral artery. J Neurosurg 1978; 49:204-228

27. Ring BA, Waddington MM. Roentgenographic anatomy of the pericallosal arteries. Am J Roentgenol Radium The Nucl Med 1968; 104:109-118

28. Saidi H, Kitunguu PK, Ogeng'O JA. Variant anatomy of the anterior cerebral artery in adult brains. Afr J Neurol Sci 2008; 27(1):97-105

29. Uchino A, Takase Y, Nomiyama K, Egashira R, Kudo S. Fenestration of the middle cerebral artery detected by MR angiography. Magn Reson Med Sci 2006; 5(1):51-55

30. Ugur HC, Kahilogullari G, Esmer AF, Combert A, Kanpolat Y. A neurosurgical view of anatomical variations of the distal anterior cerebral artery: An anatomical study. J Neurosurg 2006; 104:1-7 